\title{
Erratum to: Recombinant soluble human thrombomodulin (thrombomodulin alfa) in the treatment of neonatal disseminated intravascular coagulation
}

\author{
Akira Shirahata • Jun Mimuro • Hoyu Takahashi • Isao Kitajima • Hajime Tsuji • \\ Yutaka Eguchi • Tadashi Matsushita - Masahiro Kajiki • Goichi Honda • Yoichi Sakata
}

Published online: 29 July 2014

(C) Springer-Verlag Berlin Heidelberg 2014

\section{Erratum to: Eur J Pediatr (2014) 173:303-311 \\ DOI 10.1007/s00431-013-2155-8}

The original version of this article unfortunately contains an error in Table 1. The authors had mistakenly indicated an incorrect value in the percent columns under "Neonatal asphyxia/ HIE" and "Others". The previously erroneous values, however, do not change the conclusion of the publication. The correct version of Table 1 is presented below:

The online version of the original article can be found at: http://dx.doi. org/10.1007/s00431-013-2155-8.

\footnotetext{
A. Shirahata

University of Occupational and Environmental Health, Japan,

Kitakyushu, Fukuoka, Japan

J. Mimuro $\cdot$ H. Takahashi $\cdot$ I. Kitajima $\cdot$ H. Tsuji $\cdot$ Y. Eguchi $\cdot$

T. Matsushita $\cdot$ Y. Sakata

The Japanese Society on Thrombosis and Hemostasis Post

Marketing Surveillance Committee for Thrombomodulin Alfa,

Tokyo, Japan

J. Mimuro $\cdot$ Y. Sakata

Division of Cell and Molecular Medicine, Center for Molecular Medicine, Jichi Medical University, School of Medicine, Tochigi, Japan

H. Takahashi

Department of Internal Medicine, Niigata Prefectural Kamo Hospital,

Niigata, Japan

\section{Kitajima}

Department of Clinical Laboratory and Molecular Pathology,

Graduate School of Medical and Pharmaceutical Science, University

of Toyama, Toyama, Japan
}

\author{
H. Tsuji \\ Department of Blood Transfusion, Kyoto Prefectural University of \\ Medicine, Kyoto, Japan \\ Y. Eguchi \\ Critical and Intensive Care Medicine, Shiga University of Medical \\ Science, Shiga, Japan

\section{T. Matsushita} \\ Department of Transfusion Medicine, Nagoya University Hospital, \\ Aichi, Japan \\ M. Kajiki $\cdot$ G. Honda \\ Pharmaceuticals Sales Division, Asahi Kasei Pharma Corporation, \\ Tokyo, Japan

\footnotetext{
A. Shirahata $(\square)$

Kita-kyushu Yahata Higashi Hospital, 2-1-17 Nishi-honmachi, Yahata-higashi-ku, Kita-kyushu, Fukuoka 805-0061, Japan e-mail: a-shirahata@kitakyu-hp.or.jp
} 
Table 1 Underlying diseases causing DIC and patient characteristics

\begin{tabular}{|c|c|c|c|c|c|c|c|c|c|c|c|}
\hline \multirow[t]{3}{*}{ Patient characteristics } & & \multicolumn{8}{|c|}{ Underlying disease associated with DIC } & \multirow{2}{*}{\multicolumn{2}{|c|}{ Total }} \\
\hline & & \multicolumn{2}{|c|}{ Infections $^{\mathrm{b}}$} & \multicolumn{2}{|c|}{$\begin{array}{l}\text { Neonatal } \\
\text { asphyxia/ HIE }\end{array}$} & \multicolumn{2}{|c|}{$\begin{array}{l}\text { Hematological } \\
\text { malignancies }\end{array}$} & \multicolumn{2}{|c|}{ Others $^{\mathrm{e}}$} & & \\
\hline & & $n$ & Percent & $n$ & Percent & $n$ & Percent & $n$ & Percent & $n$ & Percent \\
\hline \multirow[t]{4}{*}{ Gestational age (weeks) } & $22-27$ & 14 & 56.0 & 0 & 0.0 & 0 & 0.0 & 1 & 6.7 & 15 & 25.0 \\
\hline & $28-36$ & 4 & 16.0 & 3 & 18.8 & 1 & 25.0 & 3 & 20.0 & 11 & 18.3 \\
\hline & $37-$ & 6 & 24.0 & 13 & 81.3 & 2 & 50.0 & 9 & 60.0 & 30 & 50.0 \\
\hline & Unknown & 1 & 4.0 & 0 & 0.0 & 1 & 25.0 & 2 & 13.3 & 4 & 6.7 \\
\hline \multirow[t]{4}{*}{ Birth weight(g) } & -999 & 14 & 56.0 & 1 & 6.3 & 0 & 0.0 & 1 & 6.7 & 16 & 26.7 \\
\hline & $1,000-1,499$ & 2 & 8.0 & 1 & 6.3 & 0 & 0.0 & 1 & 6.7 & 4 & 6.7 \\
\hline & $1,500-2,499$ & 4 & 16.0 & 4 & 25.0 & 1 & 25.0 & 3 & 20.0 & 12 & 20.0 \\
\hline & $2,500-$ & 5 & 20.0 & 10 & 62.5 & 3 & 75.0 & 10 & 66.7 & 28 & 46.7 \\
\hline \multirow[t]{3}{*}{ Type of delivery } & Vaginal & 9 & 36.0 & 8 & 50.0 & 1 & 25.0 & 8 & 53.3 & 26 & 43.3 \\
\hline & Caesarean & 15 & 60.0 & 7 & 43.8 & 3 & 75.0 & 7 & 46.7 & 32 & 53.3 \\
\hline & Unknown & 1 & 4.0 & 1 & 6.3 & 0 & 0.0 & 0 & 0.0 & 2 & 3.3 \\
\hline \multirow[t]{2}{*}{ Maternal complications at delivery ${ }^{a}$} & Yes & 8 & 34.8 & 2 & 12.5 & 2 & 50.0 & 1 & 6.7 & 13 & 22.4 \\
\hline & No & 15 & 65.2 & 14 & 87.5 & 2 & 50.0 & 14 & 93.3 & 45 & 77.6 \\
\hline
\end{tabular}

Numbers in bold are the corrected percent values

${ }^{a}$ Maternal complications at delivery: threatened labor; placenta previa, premature rupture of membranes, cephalopelvic disproportion, twin pregnancy, placental transfusion syndrome, gestational hypertension, uterine hypotonus, abnormal labor; glaucoma, ulcerative colitis, myotonic dystrophy, mumps ${ }^{\mathrm{b}}$ Infections: sepsis $(n=12)$, intrauterine infection $(n=3)$, peritonitis $(n=3)$, infection (location unknown) $(n=2)$, herpesvirus infectious disease, fungal infection, neonatal infection, urinary tract infection, necrotizing colitis

${ }^{c}$ Neonatal asphyxia/HIE: neonatal asphyxia $(n=13)$, hypoxic-ischemic encephalopathy (HIE) $(n=3)$

${ }^{\mathrm{d}}$ Hematological malignancies: acute lymphocytic leukemia $(n=2)$, myeloproliferative disorder $(n=2)$, transient abnormal myelopoiesis $(n=2$; one with Down syndrome)

${ }^{\text {e }}$ Others: small for gestational age $(n=2)$; shock; metabolic disorder; cytogenetic aberration, gastro-intestinal perforation, imperforate anus repair; hemangioma-thrombocytopenia syndrome, carbamoy phosphate synthetase deficiency, anemia neonatorum, cardiovascular collapse, fetal hydrops, cardiac failure, thrombocytopenia, hemophagocytic syndrome 